\title{
EVOLUTION OF THE MILLIARCSECOND POLARIZATION STRUCTURE OF THE BL LACERTAE OBJECT OJ 287
}

\author{
D. H. Roberts, D. C. Gabuzda, and J. F. C. Wardle \\ Department of Physics \\ Brandeis University \\ Waltham, MA 02254 USA
}

\begin{abstract}
The $\lambda 6 \mathrm{~cm}$ total intensity and polarization structures of $0 \mathrm{~J} 287$ have been determined at two epochs a year apart. At each epoch the source can be modeled as a linear structure consisting of a core and two knots at position angle $P A \simeq-115^{\circ}$. This structure expanded slightly over a year, each knot increasing its separation from the core by $\simeq 0.3$ mas, suggesting an apparent superluminal velocity of $\simeq 3.3 h^{-1} c$ for each.
\end{abstract}

\section{INTRODUCTION}

The BL Lacertae object $0 J 287(0851+202)$ is an extremely active galactic nucleus at $z=0.306$ (Miller, French, and Hawley 1978; Sitko and Junkkarinen 1985). At both optical and radio wavelengths it is violently-variable, both in intensity and polarization. Here we summarize the results of polarization-sensitive VLBI observations made at two epochs a year apart. Preliminary reports were given by Roberts and Wardle (1986, 1987); complete details may be found in Roberts, Gabuzda, and Wardle (1987).

\section{OBSERVATIONS AND DATA PROCESSING}

Polarization-sensitive $\lambda 6 \mathrm{~cm}$ observations were carried out in December 1981 and December 1982 with the Haystack, Green Bank, and Owens Valley antennas and the phased-up VLA, using the Mark III data recording system. Maps of the total intensity were made by standard hybrid mapping techniques. After correction for instrumental contamination (Roberts et al. 1984), maps of the distribution of polarized intensity $\left(P=p e^{2 i \chi}=m I e^{2 i \chi}\right.$, where $p=m I$ is the polarized intensity, $m$ the fractional linear polarization, and $\chi$ is the position angle of the electric vector on the sky) were made by referencing the cross-hand fringes to the parallel-hand fringes using the antenna gains determined in hybrid mapping, Fourier transforming the result, and performing a complex CLEAN.

\section{RESULTS}

Maps of the $I$ and $P$ distributions at the two epochs are presented by Roberts and Wardle $(1986,1987)$. We fit simple models (Table 1) to the visibility data produced by the mapping process. Requiring that the models account for the $I$ and $P$ data simultaneously led to three components, a core $\mathrm{C}$ and two knots $\mathrm{K} 1$ and $\mathrm{K} 2$. The core $\mathrm{C}$ was weakly polarized $(\simeq 4 \%)$ at each epoch; its polarization position angle changed by at least $\simeq 60^{\circ}$ between epochs. 
The inner knot $\mathrm{K} 2$ was strongly polarized at each epoch, and decreased its polarization from $\simeq 64 \%$ to $\simeq 23 \%$ between epochs. The outer knot $\mathrm{K} 1$ was only weakly polarized at each epoch, causing the predominant polarization structure to be double. The polarization position angle of each knot was roughly constant between epochs. The polarization of K2 in December 1981 is close to the maximum possible for optically-thin synchrotron radiation from electrons with a random pitch angle distribution in a homogeneous magnetic field.

Using a Hubble constant of $100 \mathrm{~h} \mathrm{~km} / \mathrm{s} \cdot \mathrm{Mpc}$ and assuming $q_{0}=0.5$, the radial motions of $\mathrm{K} 1$ and $\mathrm{K} 2$ each correspond to an apparent transverse velocity $v_{\perp} \simeq 3.3 h^{-1} \mathrm{c}$. While "two epochs do not a superluminal make," our observations are consistent with inclusion of OJ287 in the class of superluminal objects. It is interesting to note that the only other BL Lacertae object currently known to display superluminal motion, BL Lac itself, has an apparent expansion velocity of $v_{\perp} \simeq 2.4 h^{-1} c$ (Mutel and Phillips 1984). If such modest superluminal velocities are representative of BL Lacertae objects in general, this favors the view that the jets in these objects are aligned particularly closely to the observer's line of sight. The maximum of the distribution of $v_{\perp}$ with angle $\theta$ between the line of sight and the jet velocity vector occurs at $\theta=1 / \gamma$. Modest superluminal velocities, such as those observed for OJ287 and BL Lac, would occur if the BL Lacertae objects constitute a population of sources in which $\theta<1 / \gamma$. This would also be consistent with the extreme "blazar" properties of these objects (Angel and Stockman 1980).

This work would not have been possible without the assistance of Dr. Alan Rogers. We also thank the staffs of the US VLBI Network observatories and of the Haystack Mark III Processor for their help. Financial support was provided by the NSF under grants AST-82-13991 and AST-84-18636 (JFCW) and AST-83-15945 and AST-85-19529 (DHR).

Table 1: Three-Component Models for OJ287

\begin{tabular}{|c|c|c|c|c|c|c|c|c|}
\hline Component & $\alpha(\operatorname{mas})$ & $\delta($ mas $)$ & $\mathrm{I}(\mathrm{mJy})$ & $\mathrm{p}(\mathrm{mJy})$ & $\mathrm{m}(\%)$ & $\chi(\mathrm{deg})$ & $r(\text { mas })^{a}$ & $\theta(\operatorname{deg})^{a}$ \\
\hline \multicolumn{9}{|c|}{ December 1981} \\
\hline $\mathbf{C}$ & & $\cdots$ & 2910. & 98 & 3.4 & 160. & & \\
\hline K2 & -0.88 & -0.43 & 284. & 180. & 64. & 83. & 0.98 & -116 \\
\hline K1 & -2.08 & -1.20 & 243. & 6.6 & 2.7 & 65. & 2.40 & -120 \\
\hline \multicolumn{9}{|c|}{ December 1982} \\
\hline $\mathrm{C}$ & & $\ldots$ & 2830. & 129. & 4.6 & 99. &. & $\cdots$ \\
\hline K2 & -1.15 & -0.48 & 415. & 95 . & 23. & 78 . & 1.25 & -113 \\
\hline K1 & -2.55 & -0.82 & 116. & 4.0 & 3.4 & 72. & 2.68 & -108 \\
\hline
\end{tabular}

a Separation and position angle with respect to the core.

\section{REFERENCES}

Angel, J. R. P., and Stockman, H. S. 1980, Ann. Rev. Astr. Ap., 8, 321-361.

Miller, J. S., French, H. B., and Hawley, S. A. 1978, in Pittsburgh Conference on BL Lac Objects, ed. A. M. Wolfe (Pittsburgh: University of Pittsburgh), 176-191.

Mutel, R. L., and Phillips, R. B. 1984, in VLBI and Compact Radio Sources, ed. R. Fanti, K. Kellermann, and G. Setti (Dordrecht: Reidel), 117-118.

Roberts, D. H., Gabuzda, D. C., and Wardle, J. F. C. 1987, Ap. J., submitted.

Roberts, D. H., et al. 1984, in VLBI and Compact Radio Sources, ed. R. Fanti, K. Kellermann, and G. Setti (Dordrecht: Reidel), 35-38.

Roberts, D. H., and Wardle, J. F. C. 1986, in Quasars, ed. G. Swarup and V. K. Kapahi, (Dordrecht: Reidel), 141-147.

Roberts, D. H., and Wardle, J. F. C. 1987, in Superluminal Radio Sources, ed. J. A. Zensus and T. J. Pearson, (Cambridge: Cambridge University Press), 193-199.

Sitko, M. L., and Junkkarinen, V. T. 1985, P. A. S. P., 97, 1158-1162. 